\title{
The development and implementation of a
}

\author{
peer support model for a specialist mental
}

\author{
health service for older people: Lessons
}

\author{
learned
}

\section{Abstract}

Background and Objectives:

There has been a significant growth in the employment of peer workers over the past decade in youth and adult mental health settings. Peer work in mental health services for older people is less developed, and there are no existing peer work models for specialist mental health services for older people in Australia. We developed and implemented a peer work model for older consumers and carers of a specialist mental health service. This paper describes the model, outlines the implementation barriers experienced and lesson learned, and comments on the acceptability of the model from the perspective of stakeholders.

Research Design and Methods:

To ensure the development of the peer work model met the needs of key stakeholders, we adopted an evaluation process that occurred alongside the development of the model, 
informed by action research principles. To identify stakeholder preferences, implementation barriers and potential solutions, and gain insight into the acceptability and perceived effectiveness of the model, a range of methods were used, including focus groups with the peer workers, clinicians and steering committee, consumer and carer surveys, field notes, and examination of project documentation.

Results:

While the model was overall well received by stakeholders, we experienced a range of challenges and implementation barriers, in particular around governance, integrating the model into existing systems, and initial resistance to peer work from clinical staff.

Discussion and Implications for practice:

Older peer workers provide a valuable contribution to the mental health sector through the unique combination of lived experience and ageing. We recommend that models of care are developed prior to implementation so that there is clarity around governance, management, reporting lines and management of confidentiality issues. 


\section{Introduction}

There has been significant growth in the employment of peer workers in mental health settings in the United States, the United Kingdom, Australia and New Zealand over the past decade (Chapin et al., 2013; Repper and Carter, 2011). The employment of peer workers is a key component of transforming mental health services to a recovery orientation and is now considered integral to the delivery of recovery oriented services for people with mental illness, nationally and internationally (Commonwealth of Australia, 2013; Mental Health Commission, 2014). More so, integrating peer work into health systems is now at the heart of national and international reform agendas in mental health (Mental Health Commission, 2014; Gillard et al., 2013; Health Workforce Australia, 2014).

Peer work is about offering and receiving help, based on shared understanding, respect and mutual empowerment between people in similar situations (Repper, 2013; Repper and Carter, 2011). Peer work facilitates a level of understanding, support, acceptance, hope and a belief in recovery that comes through sharing personal experiences of living with and overcoming mental health issues (Commonwealth of Australia, 2013; Repper and Carter, 2011). This mutuality can help alleviate the alienation and loneliness associated with having a mental health illness, reduce symptoms and improve the wellbeing of both the people who receive it and those who provide it (Commonwealth of Australia, 2013; Jacobson et al., 2012; Miyamoto and Sono, 2012).

While the evidence base for peer work has been growing rapidly (for a review see Repper and Carter (2011); Gopalan et al. (2017)), much of the evidence is specific to early or middle 
adulthood and there is a paucity of literature regarding the peer work needs of older people (Daley et al., 2013; Chapin et al., 2013). Evidence specific to the recovery needs of older people suggests that the experience for older people is different from younger people and that this impacts on their peer work needs (Daley et al., 2013; McKay et al., 2015). For example, findings by Daley et al. (2013) suggest that older people are more likely than younger people to have a well-established sense of identity, and recovery involves reconnecting to their existing sense of self as opposed to developing a 'new' 'healthier' self. With the exception of one peer support intervention 'Reclaiming Joy' developed in the United States (Chapin et al., 2013) there is no evidence specific to the implementation and effectiveness of peer work models in older people's mental health service settings.

From early 2016, in response to increasing calls for peer work to become integrated into mental health service delivery, we developed and implemented a peer work model for older consumers and carers of the specialist mental health service for older people in Australia. This project was developed as a partnership between the public mental health service and a non-government organisation (NGO). In early 2016 this project commenced with the recruitment of eight older peer workers, of which five continued their involvement until the end of the evaluation phase, i.e. twelve months following commencement of the project. The peer workers were employed by the NGO to provide a peer work service to the Specialist Mental Health Service for Older People (SMHSOP). By early 2017 the peer work model was developed, implemented, evaluated and embedded into the service system. The peer work model includes the provision of one on one support for consumers and carers by peer workers, group work co-facilitated by peer workers, as well as broader mental health promotional activities. 
This paper provides an overview of the model, outlines the implementation barriers experienced and how these were overcome or managed, and comments on the acceptability of the model from the perspective of stakeholders. Our aim here is to guide others embarking on the development of a peer work model for older people, by outlining our specific learnings for each component of the model (recruitment, the role of peer workers, training and supervision, and governance). Given the lack of evidence in this space, the extent to which we could be guided by the existing literature in the development of a peer work model was limited. As such, to ensure the model was evidence based and met the needs of stakeholders, this project was conducted within an evaluation framework that was iterative and occurred alongside the development of the model, informed by action research principles. While some of the component of the model were pre-determined (e.g. approximate number of peer workers, governance arrangement), in as far as practicable the model was developed, or at least refined, during the implementation process, informed by the views and feedback of stakeholders.

The specific objectives of this study were to identify a) stakeholder preferences in the design of the model, b) implementation challenges and barriers as they occurred, c) ways in which to overcome or manage these barriers and d) the effectiveness or acceptability of the model from the perspective of stakeholders. The findings presented in this paper are part of a larger evaluation, and the qualitative findings specific to the views and perceptions of the peer workers themselves have been outlined elsewhere (blinded for review).

\section{Methodology}


In accordance with action research principles, this study sought to develop and implement a peer work model by working collaboratively with all stakeholders (Koshy et al., 2011). While the researchers utilised a number of methods to consult and engage stakeholders and documented the finding systematically, participants were understood as partners who actively contributed to the design of the model (Schmittdiel et al., 2010). Action research is increasingly employed in health settings as it provides a framework for clinicians to reflect on their practice in a systematic way, encouraging participants to 'own' the improvement process, and embed the service change into everyday practice (Koshy et al., 2011; Meyer, 2000). This approach is particularly suitable to studies that are concerned with both process learnings as well as outcomes (Koshy et al., 2011), as was the case for this study.

To identify stakeholder preferences, implementation barriers and potential solutions, and gain insight into the acceptability and perceived effectiveness of various component of the model, a range of methods were used, including focus groups with the peer workers, clinicians and the project's steering committee, consumer and carer surveys, field notes, and examination of project documentation (see Table 1). The data derived from these different methods were compared against each other and synthesised thematically to provide learning for each key component of the model (recruitment, the role of peer workers, training and supervision, and governance).

Table 1: Samples and data collected

Feedback from peer workers: focus groups 
Three focus groups were conducted with the peer workers throughout the evaluation period by the service researcher (first author). Each focus group was around two hours in duration. All actively engaged peer workers attended each focus group; the first focus group had eight participants and the subsequent focus groups five. While each focus group had a slightly different focus, each focus group explored the peer workers' thoughts and feelings about what is working well, things that need to change, how to make the suggested changes, and how they were personally impacted by the work. Introverted focus group participants were encouraged by interviewer during discussion to achieve a balance with the extroverted participants.

The focus groups were audio recorded, and transcribed. Inductive thematic analysis of the transcripts was conducted by the service researcher as per guidelines provided by Braun and Clarke (2006). The transcripts were read several times to identify themes and categories (Braun and Clarke, 2006). From this a coding frame was developed using NVivo 10 (Richards, 1999; Siccama and Penna, 2008), a computer based data qualitative analysis package. If new codes emerged, the coding frame was changed and the transcripts were reread according to the new structure (Braun and Clarke, 2006).

$\underline{\text { Feedback from the peer work steering committee and clinicians }}$

Analysis of project documentation and field notes maintained by the project managers

Relevant project documentation was reviewed and analysed thematically, specifically the peer work steering committee minutes. The peer work steering committee met monthly to 
oversee the development and implementation of the model and was made up of management, multidisciplinary clinicians including a psychiatrist, the service researcher, a consumer representative, and a representative from the Mental Health Coordinating Council (MHCC).

In addition, the project managers (second and third author) maintained field notes and systematically documented their observations with a focus on implementation challenges and barriers and project strengths and limitations. This included feedback received from clinician, consumers and carers. This process was used to systematically document observations from the project managers, and drawn on to describe the model, the implementation barriers and lessons learned. The field notes from both managers were combined and analysed thematically by the service researcher.

Focus groups with steering committee and clinicians

The service researcher conducted a focus group with the members $(\mathrm{N}=8)$ of the peer work steering committee six months after project commencement, which was 1.5 hours in duration. Questions focused on areas of tension in the development and implementation of the model as identified by a review of the minutes. For example, "In reviewing the minutes I noted that there are different views around the training needs of peer workers, what are some of the tensions in this space”? The focus group was audio recorded, transcribed in full and analysed thematically as per the principles outlined above.

The team of multidisciplinary clinicians participated in two focus groups, one conducted by the service researcher (first author) around six month into the project, and one conducted by 
one of the project managers (last author) towards the end of the project $(\mathrm{N}=7)$. Both focus groups were around one hour in duration, and questions pertained to perceived strengths and challenges of the project from the clinicians' perspective. At the time of the focus groups clinicians had had varying levels of exposure to the model, with some having engaged more than others. The focus groups were not audio-recorded, but a scribe took notes, including verbatim quotations. This data was analysed thematically.

\section{$\underline{\text { Feedback from consumers and carers }}$}

Feedback from consumers and carers was sought about their experiences of one on one support from a peer worker and/or participating in a group co-facilitated by a peer worker. All consumer who participated in the group program were invited to complete an anonymous feedback survey $(\mathrm{N}=30, \mathrm{n}=19)$. Participants were asked to rate a number of statements on a five point Likert scale from very much to not at all, including: is it valuable to have a peer worker co-facilitating this group; was attending the group worthwhile; and has the group impacted on the way you think about the challenges you face? Participants were also requested to list the components of the group they found most and least helpful, and what they would like to see in the future. Feedback surveys were distributed at completion of the group and returned in a sealed envelope to the project managers for analysis.

Consumers and carers who received an individual session with a peer worker $(\mathrm{N}=20)$ were invited to complete a survey designed by the peer workers themselves ( $n=7$ consumers and $\mathrm{n}=2$ carers). Participants were asked to rate a number of statements around their satisfaction with the service on a five point Likert scale from strongly agree to strongly disagree. 
Statements included: the support and encouragement I received has helped; the work done by the peer worker complemented the work of the clinician; I have a clear understanding of what peer work means; I/we have felt free to talk about my/our own experiences; peer workers have shown complete respect; the peer worker stories have given me/us hope. The survey also included a number of open ended questions around the consumers' and carers' views on strengths and limitations of the peer work service provided. Consumers and carers were given the feedback survey by their allocated clinician, who gave the completed survey to the project managers for analysis.

\section{Ethics}

Ethical approval was not required as the project was reviewed to be exempt from ethical review by a Human Research Ethics Committee and deemed as an evaluation or quality assurance Activity as per criteria set by NSW Health (2007). The project was reviewed and authorised by the delegated representative of the organisation in line with the Health Records and Information Privacy Act (HRIP Act) (2002), Ethical Considerations in Quality Assurance and Evaluation Activities developed by the National Health and Medical Research Council (NHMRC) (2014) and the National Statement on Ethical Conduct in Research (2015).

The study was conducted in accordance with the National Statement on Ethical Conduct in Research (2015). Informed consent was received from all participants. Focus group participants were provided with an information statement and signed consent forms were returned prior to the first focus group. Survey participants were informed that their responses would be used for research and evaluation purposes. While qualitative research is susceptible 
to ethical dilemmas as it is not possible to predict all questions and responses and what participants may disclose (Morse, 2007; Duncan et al., 2009), no ethical challenges were experienced.

\section{Findings and Discussion}

While we have developed a peer work model that is now well received by stakeholders and has become embedded into practice, we experienced a range of challenges and implementation barriers, in particular around governance, integrating the model into existing systems and practices and initial resistance to peer work from clinical staff.

\section{Recruitment}

In March 2016 eight peer workers were employed on a casual basis to work on average three to six hours a week. The peer work roles were advertised through a range of methods, including newspapers and a number of email lists. We recognised that some of the applicants may need support and encouragement to apply for the positions, particularly those that had been out of the workforce for some time, and as such we hosted an information/support session prior to the interviews. This session was used to provide potential applicants with context around the nature of the role and answer questions. Topics discussed included the meaning of recovery for older people, the philosophy and nature of peer work, what training will be available as part of the position, preparing resumes and addressing the criteria for the 
position.

Eleven applicants were interviewed. Interview questions focused on previous work experience, understanding of privacy, confidentiality and personal boundaries, understanding of personal recovery and how they would use their lived experience of mental illness and ageing to assist others. Three interviewees were unsuccessful and were provided with feedback and support, particularly acknowledging and respecting the stories they shared in the interview.

In terms of payment, peer work may be either paid or voluntary, strictly depending on the peer worker's individual needs and circumstances not the organisation (Repper and Carter, 2011; Commonwealth of Australia, 2009). Five peer workers requested to be paid, and three wanted to participate in a voluntary capacity. Paying peer workers proved to be more complicated than anticipated. Older people can have complex financial and income support arrangements in place (i.e. pensions and superannuation) that can be jeopardised by extra income. For example, people in receipt of the aged care pension can only work limited hours per week before their payments are affected, and they are required to report changes to their income.

I'd like to see the work not spasmodic. I'd like to see it regular otherwise it is going to be difficult for us. We have already struck one challenge in that we are dependent on a pension... We did not get the pension paid because we didn't report our income. It might seem such a simple thing to salary earners but to suddenly not have any money in the bank, it's pretty scary and that's what happens. (Peer Worker 4) 
It is critical that older peer workers have a good understating of the impact of additional income and understand income reporting requirements. We developed systems and processes to ensure peer workers were not disadvantaged by working more hours than was in their interest.

Five out of the eight peer workers initially recruited have remained actively engaged with the model. Three disengaged within the first three months of the project; one gained full time employment, one returned to teaching on a part-time basis, and one moved out of area.

\section{The peer work role}

Role ambiguity and role uncertainty inherent in peer work are commonly reported implementation barriers (Health Workforce Australia, 2014; Simmons et al., 2017; Miyamoto and Sono, 2012). To combat this, we actively engaged peer workers in defining the peer work role. Informed by the literature (in particular Health Workforce Australia (2014)), the peer workers identified three major areas of work, which have become central to the model: cofacilitated group work; individual peer work with consumers and carers; and education, advocacy and mental health promotion activities. The peer workers settled into these roles comfortably and coped well with the role ambiguity inherent to peer work as well as new projects. We attribute the peer workers' ability to cope with ambiguity and role uncertainty to their maturity, and this is explored further in (blinded for review). 


\section{$\underline{\text { Co-facilitated group work }}$}

One of the first achievements of the peer work program was the development and delivery of a recovery based group program for older people. This group was co-designed and cofacilitated by peer workers and clinicians. The group is a closed group consisting of two hour sessions once a week for four weeks, with a set program that includes the sharing of personal stories of recovery, identifying personal values and goals, and self-care activities. The group program was developed shortly after the peer workers were recruited and five groups were delivered in the first six months.

From the perspective of peer workers, co-facilitating the groups was a very positive experience.

I walked out of these sessions with a spring in my step ... I think we help people take the first step. (Peer Worker 4)

I'm a changed man since this program. I think it is life changing. The two words that I would use are life changing; life changing for the people attending and the more people that can come to these groups the better. (Peer Worker 5)

They all reacted positively. One lady sat there till the last ten minutes with her head bowed and there was some little comment that was made and she looked up all of a sudden and walked out with a smile on her face that makes it all worthwhile you see. (Peer Worker 1) 
The groups were also well received by consumers. All participants who completed the survey rated the group as worthwhile and having impacted positively on the way they think about their challenges. The majority $(75 \%)$ rated the co-facilitation of the group by a peer worker as important, and the remainder were mostly neutral. Initially a number of participants commented that the group was too rushed, and the group was changed from two weeks to four weeks. Participants highlighted the group's focus on self-care, being in a group of people with similar issues and the shared stories of recovery as most valuable.

These groups provided an opportunity for peer workers and clinicians to work together, which resulted in clinicians getting to know the peer workers. Working together and being able to observe the peer workers interact with consumers allowed the clinicians to gain confidence and trust in the peer workers' ability (as outlined later).

\section{$\underline{\text { Individual work with consumers and carers }}$}

Twenty consumers received individual peer work support, of approximately five hours each across multiple sessions. While peer workers were keen to work with individual consumers from the start of the project, this component of their role took some time to establish. Firstly, peer workers' capacity to provide one on one support to consumers had to be developed through training and supervision, and secondly, clinicians had to gain sufficient confidence in the peer workers to refer their clients. Initially clinicians were reluctant to refer clients for one on one peer support. 
I haven't got confidence in them yet. What are they doing with the consumers, they aren't clinicians so what do they do? I want to protect my consumers. There is not enough information on what they will do and how they will do it. (Clinician 1)

We don't yet know what the peer workers are capable of. We need to learn as we go. What if the peer workers cause damage to consumers and I have to pick up the pieces? (Clinician 5)

I'm worried about what the peer worker will say to my consumer and the impact it will have... Do we need to learn to let go more? We take a lot of individual responsibility for our consumers. (Clinician 2)

These reservations are consistent with a body of literature that highlights resistance from staff as a common implementation barrier (Berry et al., 2011; Simmons et al., 2017; Health Workforce Australia, 2014). In time, as clinicians got to know the peer workers and became more comfortable with the concept of peer work, they started to develop trust in the peer workers and engaged with the model (as outlined later).

The majority of consumers referred for peer support were isolated and lonely, as both peer workers and clinicians felt that this population could benefit most from peer work. Findings by Daley et al. (2013) supports this and suggest that older people who do not have established networks to draw on to reconnect to their pre-illness identity benefit most from peer work. The peer workers focussed on assisting consumers make meaningful and sustained connections with their local community, and, if appropriate, they shared their own recovery 
story. Peer workers perceive the sharing of their recovery story as beneficial to themselves as well as the consumers.

Well the clinician asked me if I mind talking about my own experience and I said I love talking about it. It still does me good to talk about it... I could talk about it all day... All those decades I never talked to anyone about it; not one word for 65 years. Letting it out helps you and it helps them strangely enough. (Peer Worker 2)

Communication has really changed and the peer support worker is somebody who is going to talk with them and to me that would be hugely beneficial to somebody who is feeling very isolated, even from their family and loved ones because they are pushed aside when their old. (Peer Worker 4)

I see our role not as being people who give them fast answers or any answers for that matter, but our role is to give them some hope, give them an idea that there are answers out there ... and secondary there are people out there who care about their condition and those conditions generally. I see myself doing that in two ways, one is sitting them down and letting them talk about it... seeing where that leads ... following them wherever they go and the second way is to share a little of my own experience with them and say I understand what you're feeling, I understand where you are coming from and I understand the journey that is in front of you. But I'm not going to tell them how to solve that problem because I'm not a professional. (Peer Worker 1)

In addition to clinicians' initial reluctance to refer consumers for peer support, we 
experienced a number of other challenges, in particular around the sharing of information between peer workers and clinicians about consumers. Peer workers expressed a need for more communication from clinicians about what is expected from the peer work/consumer contact, more direction as to the reasons for referral and a need for peer workers to contribute to the consumers care plan with the clinician. To enhance communication, we developed processes and procedures that stipulate clinicians' and peer workers' responsibilities in terms of the sharing of information, specifically: clinicians and peer workers have a joint session with consumers referred to peer work to develop a care plan conjointly; clinicians have a responsibility to provide peer workers with information regarding the specific recovery needs of the consumer; and following each contact with a consumer peer workers record the contact on a progress note and share the content of the session verbally with the consumer's allocated clinician.

\section{Education, advocacy and mental health promotion activities}

Peer workers delivered numerous presentations about their recovery journey and role as peer workers at a wide variety of forums, conferences, workshops, consultations and professional development days. The peer workers have a particular passion for advocacy and educating community members and service providers about 'the double whammy of experiencing old age and mental illness'. The main challenge for peer workers in this area was keeping up with the demand as this is only one component of their role.

\section{Peer Work Training and Supervision}




\section{$\underline{\text { For peer workers }}$}

To develop the peer workforce all peer workers were offered access to the Certificate IV in Mental Health Peer Work qualification to be completed over their first year of employment. The Certificate IV is a recognised qualification endorsed by the National Skills Standard Council and delivered by the Mental Health Coordinating Council (MHCC) (Mental Health Coordinating Council, 2016). The Certificate IV has a total of 16 days face to face training along with a range of assessment tasks.

While we encouraged peer workers to complete this Certificate, this was not mandatory. Although we appreciate the importance of endorsed qualifications to build the capacity of the peer workers (Shears and Ramon, 2012; Mental Health Coordinating Council, 2016), in recognition of the peer workers' ages and level of maturity (see blinded for review) as well as the debates around the professionalization of peer work, we decided some flexibility around training expectations was required. There is ongoing debate in the peer work literature around the question of whether peer workers are to remain 'befrienders', para-professionals or as a new workforce that offers specialist interventions (Shears and Ramon, 2012). While some argue peer workers are 'professionals' that need professional training (Mental Health Coordinating Council, 2016), others argue that the peer worker-consumer relationship should remain flexible and informal to avoid perpetuating the power imbalances inherent in clientconsumer relationships (Mead et al., 2001).

All five peer workers completed the face to face components of the training, and four completed all components, including assessments. While the peer workers are generally 
positive about the more practical aspects of the Certificate IV and the learning it has provided, they described some elements as "not targeted to an older peer workforce", "overwhelming", and "overly academic".

On the first day I had a brain drain but you know I hadn't studied since I was at work and then the second day was good, I was relaxed. (Peer Worker 2)

I found it hard going to be quite honest to start with but then I started to get into the groove so the answer is I think it's a very thorough complete course. (Peer Worker 3)

It's a large mountain to climb because we spent about 14 or 16 hours down there and I've spent much more time doing the homework and trying to get the answers of those questions you have got to search. I'm not complaining about that, I'm enjoying it, doing the work gets the old grey cells moving. It's been years since I've done that. (Peer Worker 5)

While the intensity of the Certificate IV was at times overwhelming, it played a role in overcoming staff concerns. The peer workers required a significant level of academic support to complete the assessment tasks. A number of clinicians were engaged to provide this support through one on one consultation and/or a monthly peer work meeting where assignment questions were brainstormed as required. As such, the clinicians became familiar with the training material, which alleviated some concerns.

They are doing a lot of study and training. I've seen their course materials and it took 
me a long time to read. They are being suitably trained. (Clinician 6)

Clinicians commented that knowing that the peer workers were receiving professional training helped increase their confidence in the peer workers. While as managers we struggled to make formalised peer work training mandatory for all peer workers, the observation that formalised training may help alleviate resistance from clinicians is a consideration.

Another important strategy used to enhance peer work capacity was supervision. The literature consistently emphasises that training on its own is not enough and needs to be paired with supervision (Repper, 2013; Orwin, 2008). Some scholars argue that effective supervision is the single most important element in successful peer work (Orwin, 2008). Supervision creates an opportunity for peer workers to develop skills, knowledge and expertise and gain confidence that any difficulties they experience are not unique to them and can be overcome (Repper, 2013). The peer workers received monthly group supervision and were allocated a clinician/manager as a mentor, which they engaged with enthusiastically.

\section{$\underline{\text { For clinicians }}$}

Repper and Carter (2011) recommend that clinicians attend the same training as peer workers to ensure they understand the role of peer workers and the training they receive, and our experiences support this recommendation. It is common for mental health staff to not fully understand the purpose and nature of peer work, and clinicians need support and training to overcome this (Simmons et al., 2017; Mental Health Commission, 2016). We did not initially 
prepare clinicians adequately and experienced resistance. While we had provided clinicians with a one day training session around recovery oriented practice and peer work, this was insufficient to elicit commitment. To overcome resistance, staff received addition training specific to peer work rather than recovery more broadly, outlining the nature of peer led, nonclinical recovery interventions; the philosophical underpinnings of peer work; the benefits of peer work for consumers, services, and peer workers themselves; and an overview of the training the peer workers were undertaking. This additional training, combined with exposure to the peer workers and the quality of their interactions with consumers, helped lessen resistance and strengthen clinician engagement with the model.

\section{Governance}

As noted, the peer workers were employed by an NGO to provide a peer work service to SMHSOP consumers and carers. The decision to deliver a peer work service to consumers of a public mental health service through a partnership with an NGO was a practical one. Firstly, the NGO already had a peer workforce and significant experience in this space, and a partnership allowed us to draw on this expertise. NGOs have taken the lead in terms of the engagement of peer workers well before public mental health services, locally as well as nationally and internationally (Repper and Carter, 2011). Secondly, a partnership with an NGO as the official employer avoided a range of bureaucratic barriers inherent to employing peer workers within government organisations. A study by Coates and Howe (2015) about the experience of developing a youth participation model in a public mental health service in Australia reported difficulties recruiting to youth participation positions because of a range of bureaucratic delays, in particular because of confusion around organisational right, or lack 
thereof, to discriminate based on age (as these positions were youth specific). As for the current project we wanted to recruit older peer workers, we were concerned we'd face similar barriers. Furthermore, as government employees, the peer workers would have been required to complete extensive mandatory training that, given they only worked around three hours each week, would consume a large part of their available hours.

While this partnership has been positive and collaborative, and may have avoided some bureaucratic challenges, it has posed a number of unanticipated challenges. From the perspective of peer workers, it was confusing, at least initially. On a day to day basis they work closely with SMHSOP clinicians and management, even though they are employed by the NGO. In the early stages of the project, peer workers consistently expressed that they were confused around the governance and reporting lines. The peer workers identified very closely with SMHSOP but not the NGO. SMHSOP exacerbated this perception by often being the go-between for the peer worker with the NGO; in hindsight it would have been preferable for the peer workers to liaise directly with their official employer around certain issues (i.e. ill-health, timesheets, etc.).

Another challenge regarded the sharing of client information with peer workers, and processes for the exchange of information between the two services were underdeveloped at the commencement of the project. While we have agreements in place that allow us to share client information with NGOs, and Health services commonly work in partnership with the NGO sector, we were concerned that some consumers and carers may not realise that the peer work service was technically not provided by the public mental health service. To overcome this we introduced a formalised consent process that stipulates that consumers are provided 
with details about the peer work service and sign a consent form prior to being referred for peer support. While the partnership arrangement added a layer of complexity around the sharing of information, tensions around the level of access peer workers should have to client information is not unique to this model and are commonly reported in the literature (Health Workforce Australia, 2014; Gillard et al., 2013; Berry et al., 2011; Simmons et al., 2017; Repper and Carter, 2011).

A key learning for the project was to ensure reporting lines and roles and responsibilities for each organisation are clearly articulated in standardised procedures and regularly communicated and reinforced. While SMHSOP and the NGO now have entered into a formal contract arrangement, we believe that this should have been in place before the peer workers were recruited.

As an outcome of this project, we recommend that models of care are developed prior to implementation, so that there is clarity around governance, management, reporting lines and the way in which confidentiality and privacy issues are to be managed. For the current project, the model of care was developed concurrently with the implementation of the model and while this approach is effective in considering the views of stakeholders in the development of a model, it carries with it an increased risk that policy and procedural requirements are underdeveloped prior to model implementation.

\section{Stakeholder feedback}

From the perspective of peer workers, analysis of focus group data indicates that peer work is 
a rewarding experience with many benefits, in particular: the opportunity for workforce participation and learning later in life; the development of new friendships and supports with other older peer workers; the joy of observing consumer and carers gain from the support provided by the peer workers; and support in terms of their own ongoing recovery.

I didn't think I'd be joining the workforce at my age... It's great to be learning at this age. (Peer Worker 3)

Some of us have become very good friends. .. [We] open up to each other and we understand each other...I wouldn't ring up my family and tell them anything like how I feel so we can really help one another. (Peer Worker 2)

The greatest highlight is seeing the positive responses from the consumers... They seem to brighten up and really connect. It's making me feel young again. (Peer Worker 5)

Being a peer worker has helped me so much. I'm a different person. Even my kids say, mum this is the best thing that's ever happened to you. 'It's changed you so much, we know you now. (Peer Worker 2)

From consumers' perspective, analysis of the feedback indicates that the peer workers offered a sense of hope for recovery consistent with the philosophy of peer work. With one exception, all consumers and carers agreed or strongly agreed with all of the statements on the individual peer work survey, rating their experience as very positive. In terms of qualitative feedback, comments such as "the peer workers are an inspiration", "they gave 
me hope"; "I liked listening to the peer workers experience of recovery"; "'they showed me a way out"; "I can tell her things that I can't say to others” were common. One consumer was less positive and commented that the peer worker might need support himself. Due the nature of mental illness, peer workers can relapse. As such, this observation was taken seriously and prompted us to strengthen processes and procedures around how to support peer workers if they were to become unwell. The peer worker in question did indeed need and received additional support.

While only two carers provided feedback by completing the survey, this feedback was positive.

I felt so much support, I'm no longer alone. (Carer 1)

This week he was so much more animated and making plans for a holiday. I'm positive your talk with him had a huge impact and it pushed him around the corner, he is actually quoting you!!! Your advice and support was fabulous and I look forward to seeing you again. (Carer 2)

Clinician feedback, while overall positive, was more cautious. As noted, while clinicians were initially reluctant to engage with the model, by the end of the evaluation period they worked collaboratively with peer workers and increasingly referred consumers for individual peer support.

She [the peer worker] has achieved more with the consumer in a few weeks than I have 
been able to achieve in two years. (Clinician 2)

I cannot express my appreciation enough for the wonderful support [the peer worker] have given to my clients... Myself and the treating team have had a struggle all this year to support [the client] to recover from depression. This week he was so much better and making plans for a holiday. (Clinician 3)

They are a lot higher level than previous peer workers I've been involved with, they are not token, they are really involved. (Clinician 4)

The strength of peer workers is lived experience. I see a dramatic change when consumers interact with the peer workers and how much it meant to them knowing that others have experienced mental illness. It's a relief off their shoulders, they are very good at just listening. Feedback from consumers is always consistent. "They are empathic, they understand, they know exactly what it's like, they see hope, it's so powerful”. (Clinician 6)

I attend the meetings with the peer workers and I've got to know them, I now feel comfortable referring to them... I really have faith in these people. They have had a lived experience. (Clinician 1)

The consumer said to me, “the clinician, you don't understand, you've never had depression but the peer worker talked about his experience of taking medication ... and that was great”. (Clinician 5) 
While the feedback is positive and the model has become, by and large, embedded into the health system, it has not been without implementation barriers and key learnings in all components of the model.

\section{Conclusion}

We describe a peer work model for older people, and outline key lessons learned during implementation and how barriers were overcome or managed. A key implementation challenge was staff resistance to peer work; to manage this and engage clinical staff in peer work, we actively engaged clinicians in the development of the model, and provided appropriate training around the purpose of peer work. The most powerful change agent for clinicians in terms of embracing peer work was their experience of working with peer workers and directly observing their unique skillset and insight when working with consumers and carers. We also experienced a number of operational issues in particular around governance and the sharing of client information, and services embarking on peer work need to identify the most appropriate strategy to engage peer workers, directly or as a partnership.

This paper also comments on the overall acceptability or effectiveness of the model from the perspective of stakeholders, and indicates that despite a range of implementation barriers, the model has been well received by peer workers themselves, clients and carers, as well as clinicians. Our findings contribute to the limited evidence base specific to the development 
and implementation of peer work models for older people, particularly in relation to process and practice outcomes. 


\section{References}

Berry C, Hayward M and Chandler R. (2011) Another rather than other: experiences of peer support specialist workers and their managers working in mental health services. Journal of Public Mental Health 10: 238-249.

Braun V and Clarke A. (2006) Using thematic analysis in psychology. Qualitative Research in Psychology 3: 77-101.

Chapin R, Sergeant J, Landry S, et al. (2013) Reclaimimg Joy: Pilot Evaluation of a Mental Health Peer Support Program for Older Adults Who Receive Medicaid. The Gerontologist 53: 345-352.

Coates D and Howe D. (2015) Integrating a youth participation model in a youth mental health service: Challenges and lessons learned Child and Youth Services.

Commonwealth of Australia. (2009) Fourth National Mental Health Plan: An agenda for collaborative government action in mental health 2009-2014. Australian Government, Department of Health and Ageing.

Commonwealth of Australia. (2013) A National Framework for Recovery-Oriented Mental Health Services - Policy and Theory. Australian Health Ministers Advisory Council.

Daley S, Newton D, Slade M, et al. (2013) Development of a framework for recovery in older people with mental disorder. International Journal of Geriatric Psychiatry: 522-529.

Duncan R, Drew S, Hodgson J, et al. (2009) Is my mum going to hear this? Methodological and ethical challenges in qualitative health research with young people. Social Science \& Medicine 69: 1691-1699.

Gillard S, Edwards C, Gibson SL, et al. (2013) Introducing peer worker roles into UK mental health service teams: a qualitative analysis of the organisational benefits and challenges. BMC Health Services Research 13.

Gopalan G, Lee SJ, Harris R, et al. (2017) Utilization of peers in services for youth with emotional and behavioral challenges: A scoping review Journal of Adolescence 55: 88-115.

Health Records and Information Privacy Act. (2002) NSW Legislation No 71. Available at: http://www.legislation.nsw.gov.au/\#/view/act/2002/71/full.

Health Workforce Australia. (2014) Mental Health Peer Workforce Literature Scan.

Jacobson N, Trojanowski L and Dewa CS. (2012) What do peer support workers do? A job description. BMC Health Services Research 12.

Koshy E, Koshy V and Waterman H. (2011) Action Research in Healthcare, London.

McKay R, McDonald R, Lie D, et al. (2015) Reclaiming the best of the biopsychosocial model of mental health care and "recovery" for older people through a 'person-centred approach. Australas Psychiatry 20: 492-495.

Mead S, Hilton D and Curtis L. (2001) Peer Support: A Theoretical Perspective. Psychiatric Rehabilitation Journal, 25.

Mental Health Commission. (2014) Living Well: A Strategic Plan for Mental Health in NSW. Sydney: NSW Mental Health Comission,.

Mental Health Commission. (2016) Employer's guide to implementing a peer workforce: Mental Health Commission, Peer Work Hub: Growing the Mental Health Workforce.

Mental Health Coordinating Council. (2016) Mental Health Peer Work Qualification Development Project. Available at: http://www.mhcc.org.au/sectordevelopment/workforce-development/peer-work-qualification-project.aspx.

Meyer J. (2000) Using qualitative methods in health related action research. British Medical 
Journal: 178-181.

Miyamoto Y and Sono T. (2012) Lessons from Peer Support Among Individuals with Mental Health Difficulties: A Review of the Literature. Clinical Practice and Epidemiology in Mental Health 8: 22-29.

Morse JM. (2007) Ethics in action: ethical principles in doing qualitative health research. Qualitative Health Research 17: 1003-1005.

National Health and Medical Research Council. (2014) Ethical Considerations in Quality Assurance and Evaluation Activities. Available at: https://www.nhmrc.gov.au/_files_nhmrc/publications/attachments/e111_ethical_consi derations in quality assurance 140326.pdf.

NSW Health. (2007) Human Research Ethics Committees - Quality Improvement \& Ethical Review: A Practice Guide for NSW. Document number GL2007_020, North Sydney: NSW Health.

Orwin D. (2008) Thematic Review of Peer Supports Literature review and leader interviews In: Commissioned by the Mental Health Commission Wellington NZ, July 2008 (ed).

Repper J. (2013) Peer Support Workers: a practical guide to implementation - Implementing Recovery through Organisational Change. Centre for Mental Health and Mental Health Network, NHS Confederation 2013.

Repper J and Carter T. (2011) A review of the literature on peer support in mental health services. Journal of Mental Health 20: 392-411.

Richards L. (1999) Using NVivo in qualitative research Thousand Oaks, CA: Sage Publications.

Schmittdiel J, Grumbach K and Selby J. (2010) System-Based Participatory Research in Health Care: An Approach for Sustainable Translation Research and Quality Improvement. Annals of Family Medicine 8: 256-259.

Shears J and Ramon S. (2012) Peer support workers: A critical analysis of an innovation in mental health. Dialogue in Praxis A Social Work International Journal. 1: 71-87.

Siccama CJ and Penna S. (2008) Enhancing Validity of a Qualitative Dissertation Research Study by Using NVivo. Qualitative Research Journal 8: 91-103.

Simmons M, Coates D, Batchelor S, et al. (2017) The CHOICE Pilot Project: Challenges of implementing a combined peer work and shared decision making program in an early intervention service. Early Interv Psychiatry. 